\title{
Data Registration, Match, and Model Component Coupling
}

\author{
Shujia Zhou* \\ NASA Goddard Space Flight Center \\ $1-301-286-8227$ \\ shujia.zhou@gsfc.nasa.gov
}

\author{
Carlos Cruz* \\ NASA Goddard Space Flight Center \\ 1-301-286-3716 \\ ccruz@pop600.gsfc.nasa.gov
}

* Employee of Northrop Grumman Corporation

\begin{abstract}
A coupling toolkit has been developed to reduce the complexity of model component coupling, in particular among hierarchical model components. The toolkit provides the services of data registration, data matching, data filtering, and model component coupling. In addition, it can generate diagrams to reveal the "producer"-to-"consumer" relations among the components. We have tested this toolkit with the operational NASA Goddard Earth Observing System (GEOS-5), which is built on the Earth System Modeling Framework (ESMF) and consists of several-level Earth system components.
\end{abstract}

\section{Keywords}

Framework, component, coupling, data registration, data match

\section{INTRODUCTION}

The component technology is being used to couple model components from different organizations $[1,2]$, and decompose a complex application, such as a combustion model $[3,4]$.

One component-based framework is the Earth System Model ing Framework (ESMF), which attempts to standardize Earth system models and their couplings $[5,6,7]$. There are two kinds of ESMF components. One is the Gridded Component for a model, and the other is the Coupler Component for coupling two model components. They have no differences except the name at this time. From the perspective of component coupling, an ESMF Grid Component has the following features:

- An ESMF component has three standard interfaces, ESMF_GridCompInitialize(...),

ESMF_GridCompRun(...),

ESMF_GridCompFinalize(...).

- User's versions of these three routines are provided through ESMF_SetEntryPoint and linked to these three standard interfaces with ESMF_SetService(...) (see Fig. 1).

- An ESMF component has only one import and one export state of ESMF_State data type (see Fig. 1).

- An ESMF coupler is used to exchange data between two sibling components (Fig. 2).

- An ESMF_State can be nested to support multiple model component coupling (see Fig. 3).

- An ESMF component can exchange data with multiple components through multiple couplers (see Fig. 3).
With ESMF, there are essentially 3 operations for model component coupling:

- Match the name of an export variable of a "producer" component with the name of an import variable of a "consumer" component.

- Transport the matched export variable to the "consumer" component.

- Transform the matched export variable as required by the "consumer" component, such as regridding.

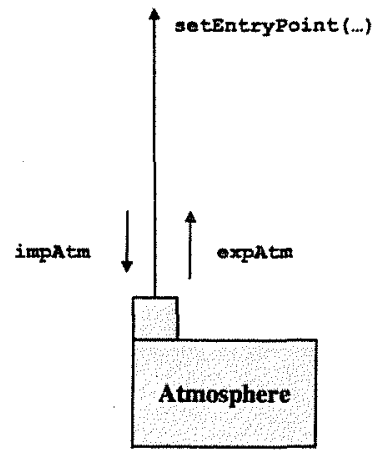

Figure 1 Schematic of an ESMF component.

To couple two model components such as atmosphere and ocean, an ESMF coupler is required to handle the data transfer for an export state (e.g., expAtm) to an import state (e.g., impAtm). Note that this coupler is located at the same level as the two model components to be coupled and these two model components are siblings, that is, they have the same parent (see Fig. 2). For the case where the component with export variables is in the same level as the component with import variables, it is straightforward for a coupler to perform the data transfer and transformation, such as regridding. However, it could be very challenging when coupling is among hierarchical components. For example, if $u$ and $v$ (west-to-east and south-to-north wind speeds) of a dynamics model component are exported to an ocean component, these two variables have to be packed into expDyn, the export state of the dynamics model component (dyn). expDyn is then passed to expAtm through expSdyn (the export state of the sdyn model component) if the feature of nested ESMF states is for data transportation (see Fig. 4). Considering the fact that each export state can contain tens of variables, it is clear that implementing 
this kind of coupling manually can be quite challenging. Therefore, a tool to reduce coupling complexity is highly desired.

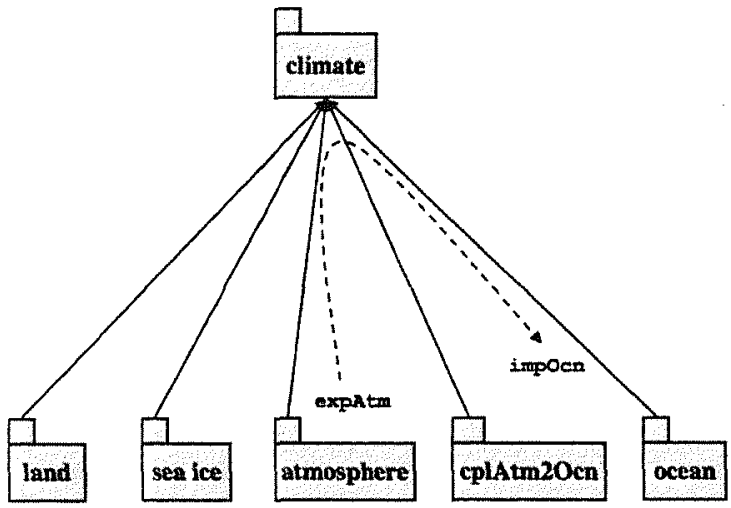

Figure 2 Illustration of coupling between two model components through ESMF. The solid line designates the hierarchical relationship while the dash line shows the data flow.

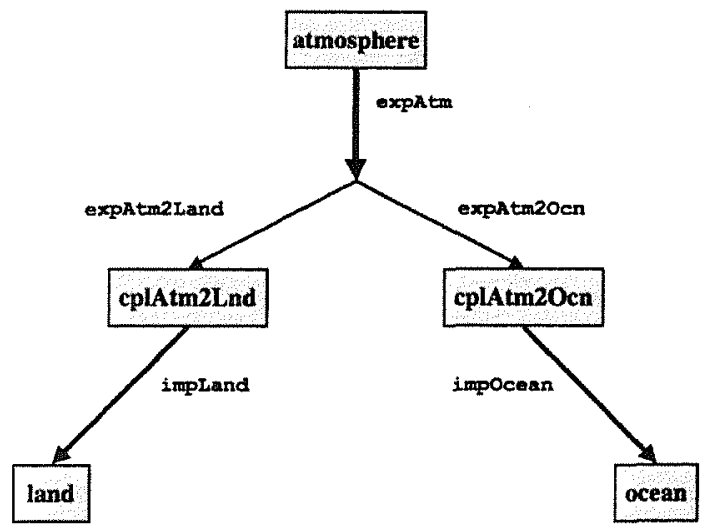

Figure 3 Illustration of a model component coupling with two model components through nested ESMF export states. The arrow designates the data flow.

\section{DESIGN}

Since ESMF provides a standard way of model component coupling for Earth system models, we outlined a generic coupler to couple model components if model components use a standard naming convention such as CF for their export and import variables [8]. That design has been implemented with MPI and successfully used in coupling an atmospheric model, WRF [9], with an ocean model [10]. However, that version needs to be augmented to deal with hierarchical components such as GEOS-5. In this paper, we present the augmented design with one implementation. Essentially, there are 4 parts: data registration, data matching, data filtering, and a code generator.

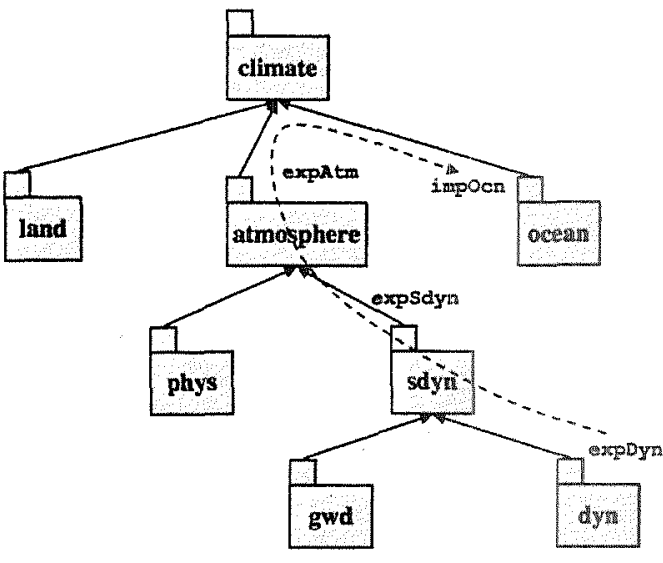

Figure 4 Illustration of coupling between two model components where there is a hierarchical relationship.

\subsection{Data registration}

For a component to interact with other components, it needs to provide the following essential information:

- component name

- import variable names

- export variable names

- parent component name in the case of hierarchical components

The auxiliary information can be added for describing data such as physical unit and dimension.

\subsection{Data matching}

Since the essential registered data are in the form of a string, data matching is string manipulation among registered components of all the levels. The output of data matching is the directional pair relationship from a producer component to a consumer component where at least the name of one export variable matches the name of one import variable. The matched data information can be used to reveal the ultimate source of exported variables.

\subsection{Data filtering}

Since an ESMF coupler is only for coupling sibling components, the coupler will be only created between two components that have the same parent. So the hierarchical positions of components will be used to determine whether a coupler is created between two components that have the directional pair relationship revealed through data matching.

\subsection{Code generator}

Since the code structures and algorithms of model components and their transformation requirement (e.g., regridding) vary considerably from each other, we believe that the intrusion to the model code would be minimized by generating the code related to ESMF coupling. This approach also allows a user to customize the coupling code to satisfy his or her specific requirements. So, 
our generic coupler toolkit will generate the stub code related to coupling for ESMF components and couplers.

We chose to perform the transformation operation only at the coupler. So, the data transporting from the source component of the matched export variable to the coupler can be done through redirecting the pointers. In that way, there is no need to include this variable (e.g., $u$ or $v$ ) in the export state of the components along the path (e.g., sdyn in Fig. 4). This avoids the potential complexity of using nested ESMF states for coupling between several-level hierarchical components.

\section{IMPLEMENTATION}

Since significant operations are string manipulation, we chose $\mathrm{C}++$ for implementation.

For the ease-to-use purpose, the format of a plain text file with a simple rule is used as an input. XML technology could be deployed if the complexity of input options increases considerably.

The output is the stub codes of ESFM components and couplers with the import and export states filled only with the matched variables. In addition, the directional export-to-import relations of components are written into a file and can be visualized with the graph visualization software, Graphviz [11].

\section{RESULTS}

We tested our generic coupler toolkit with GEOS-5 which is the operational NASA Goddard Earth Observing System built on ESMF and consisting of several-level Earth system components. [12]. One example of an input file is the portion for the AGCM component of GEOS-5:

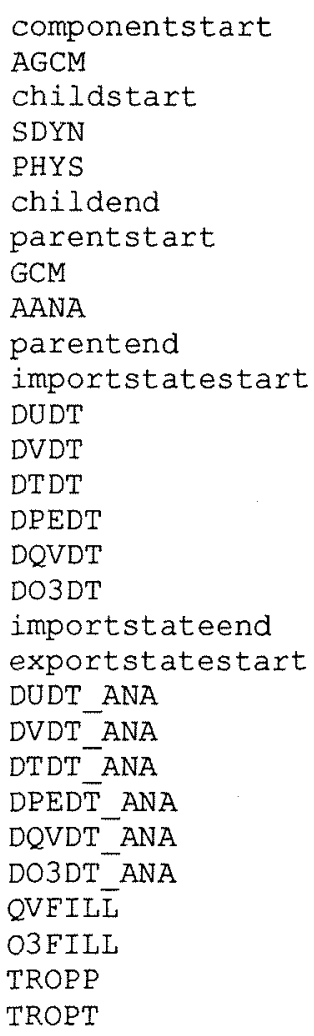

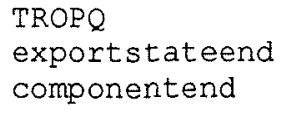

To add more components, a user can append the corresponding data into this file. Note that the names of components, import, and export variables should be consistent among all the registered components. In the case of cross-organization model component coupling, the naming convention such as $\mathrm{CF}$ or similar is likely the choice, but our tool does not depend on it.

The portion of the generated code for AGCM component is as follows:

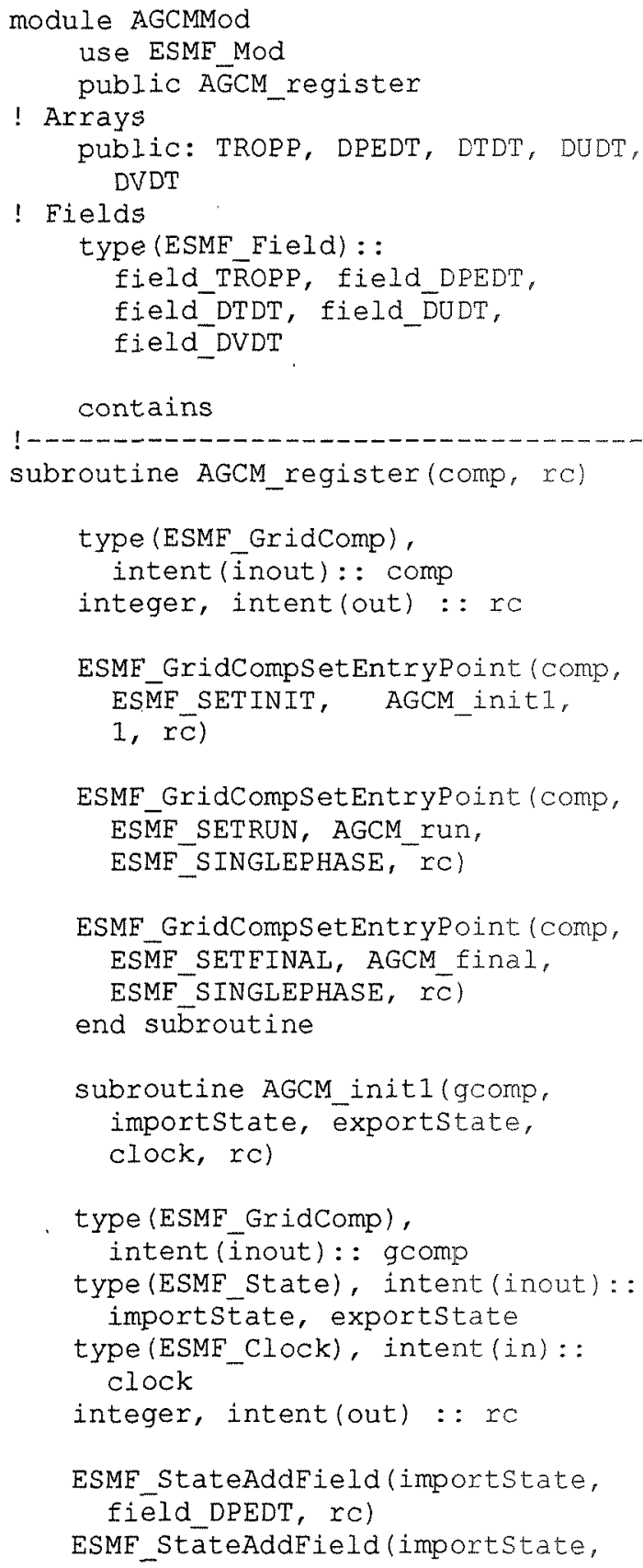




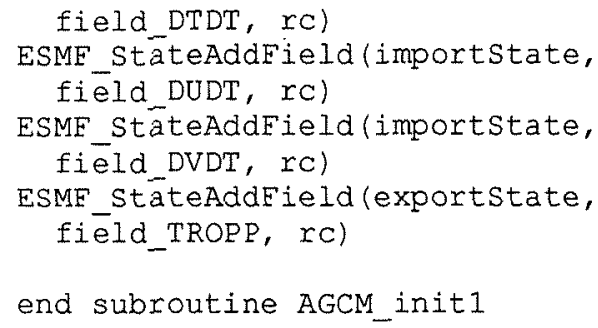

The generic coupler toolkit can also be used to generate a diagram to reveal the component relationship. Fig. 5 shows the hierarchical component relationship of GEOS-5. Note that the arrow designates the hierarchical relationship. However, that relationship is more or less the one from the perspective of "composition" rather than "inheritance" supported in an Object Oriented Language such as $\mathrm{C}++$ and Java.

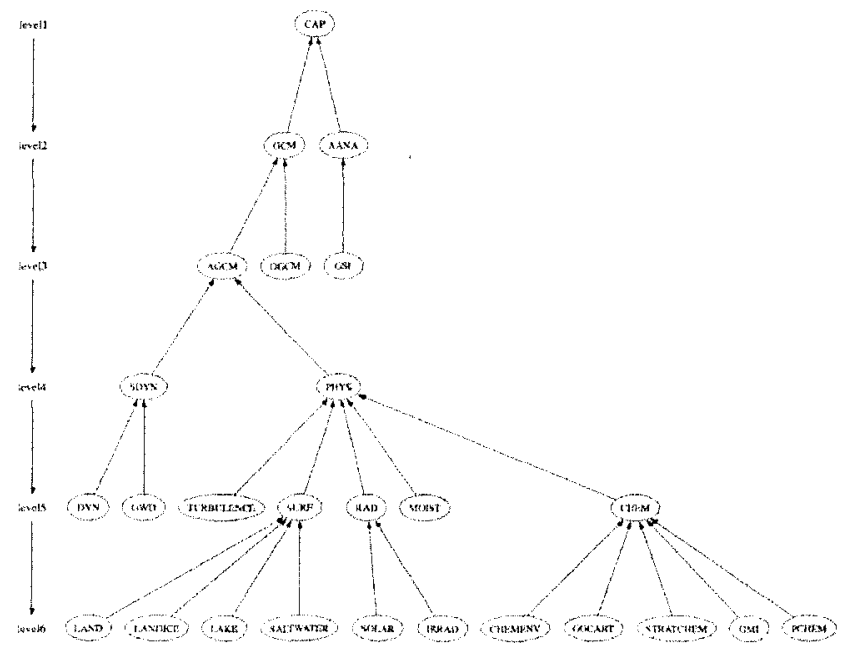

Figure 5 The diagram of the hierarchical component structure in GEOS-5.

Moreover, the generic coupler toolkit can create the export-toimport relations among all the registered components or the selected one with the rest of components. The corresponding matched variables are also written into a file for detailed analysis. Fig. 6 shows all the export-to-import relations of SURF component with other components of GEOS-5. The line with an arrow connects the component with at least one matched name of export variable to the component with the matched import variable. This kind of relationship can be very helpful for an outsider to couple a component with GEOS- 5 and understand how a new export variable impacts the scientific results of other components in GEOS-5.

Since the components of TURBULENCE, SURF, RAD and MOIST are siblings (whose parent is PHYS) as shown in Fig. 5, the couplers should be used in the case of data exchange. As shown in Fig. 6, SURF has the matched export-to-import variables with the components of TURBULENCE, RAD, and MOIST. So the couplers are needed to exchange those variables. In the case that there is no need for transformation, the export variables can be passed to the matched import variables through reassigning the pointer.

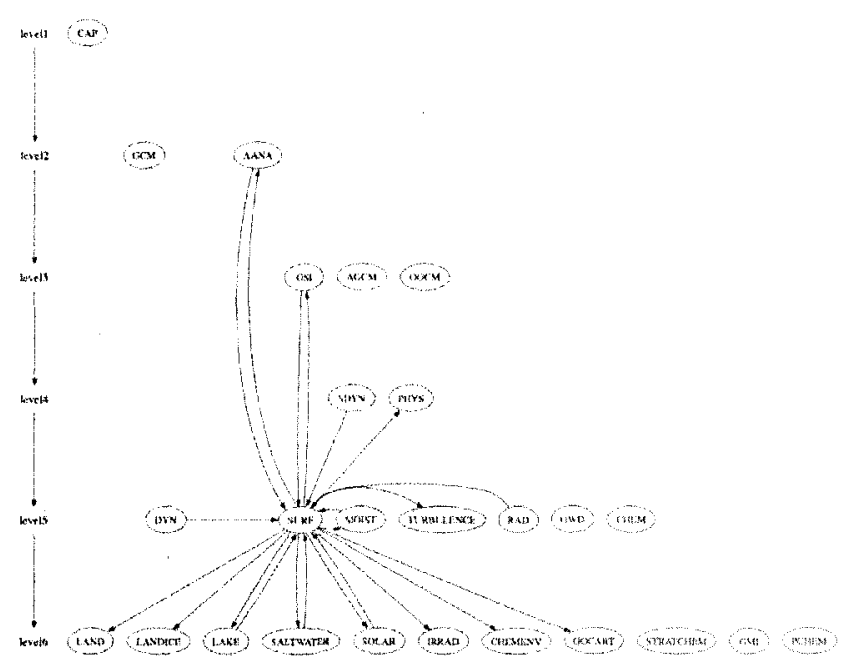

Figure 6 The export-to-import relationships of the SURF component with others in GEOS-5. Note that the arrow designates the data flow from export variables to an import variable.

\section{SUMMARY}

As shown in Figures 5 and 6, the coupling among hierarchical components can be very challenging. Our augmented design and implementation has addressed those issues in data matching and hierarchical component structure. However, further refinement is needed after more coupling applications are analyzed, in particular where transformation is involved. In addition, it is challenging to balance standardization for facilitating model component coupling with the easy-to-adopt requirement since it could take significant time and effort to adapt those applications consisting of many components for new standards. Nevertheless cross-organization model coupling is the ongoing trend and can be fruitful scientifically and financially. Our generic coupler toolkit is developed to reduce the complexity for those models to adapt such a trend.

\section{ACKNOWLEDGMENTS}

This project is supported by the NASA Earth Science Technology Office Computational Technologies Project. We would like to thank Joseph Spahr, Weiyu Yang, and Tom Clune for helpful discussions and Jarrett Cohen for editing the manuscript

\section{REFERENCES}

[1] S. Zhou, "Coupling Climate Models with Earth System Modeling Framework and Common Component Architecture," Concurrency Computation: Practice and Experience, 18203 (2006)

[2] S. Zhou et al., "Cross-Organization Interoperability Experiments of Weather and Climate Models with the Earth System Modeling Framework," Concurrency and Computation: Practice and Experience, 19583 (2007). 
[3] L.C. McInnes, B.A. Allan, R. Armstrong, S.J. Benson, D.E. Bernholdt, T.L. Dahlgren, L.F. Diachin, M. Krishnan, J.A. Kohl, J.W. Larson, S. Lefantzi, J. Nieplocha, B. Norris, S.G. Parker, J. Ray, and S. Zhou, "Parallel PDE-Based Simulations Using the Common Component Architecture," an invited chapter in the book "Numerical Solution of Partial Differential Equations on Parallel Computers," A. M. Bruaset, P. Bjorstad, and A. Tveito, editors, published by Springer-Verlag 2006.

[4] Common Component Architecture, http://www.ccaforum.org

[5] Earth System Model Framework, http://www.esmf.ucar.edu

[6] C. Hill, C. DeLuca, V. Balaji, M. Suarez, A. da Silva, and the ESMF Joint Specification Team, "The Architecture of the Earth System Modeling Framework," Computing in Science and Engineering, Volume 6, Number 1, 2004.

[7] Collins, N., G. Theurich, C. DeLuca, M. Suarez, A. Trayanov, V. Balaji, P. Li, W. Yang, C. Hill, and A. da Silva, "Design and Implementation of Components in the Earth
System Modeling Framework," International Joumal of High Performance Computing Applications, Fall/Winter 2005.

[8] S. Zhou and J. Spahr, "A Generic Coupler for Earth System. Models," Proceedings of the Parallel CFD 2005 Conference, College Park, MD, U.S.A. (May 24-27, 2005) (Parallel Computational Fluid Dynamics: Theory and Applications, Edited by A. Deane et al., Elsevier B.V., page 187-193).

[9] The Weather Research and Forecasting (WRF) Model, http://www.wrf-model.org

[10] Joseph Spahr, private communication

[11] Graph visualization software (Graphviz), http://www.graphviz.org

[12] The NASA Goddard Earth Observing System Model, Version 5 (GEOS-5), http://gmao.gsfc.nasa.gov/systems/geos5/index.php 